\title{
Experimental Study on Rotor Dynamic Lift Determination for Agricultural Unmanned Aerial Vehicle (AUAV)
}

\author{
Jing Zhang \\ College of Engineering \\ China Agricultural University \\ Beijing, China \\ cathy64882584@163.com \\ Du Chen * \\ College of Engineering \\ China Agricultural University \\ Beijing, China \\ tchendu@cau.edu.cn \\ * Corresponding Author \\ Shumao Wang \\ College of Engineering \\ China Agricultural University \\ Beijing, China \\ wangshumao@cau.edu.cn
}

\author{
Liguo Wei \\ Chinese Academy of Agricultural Mechanization \\ Sciences \\ Beijing, China
}

\author{
Quan Jia \\ Chinese Academy of Agricultural Mechanization \\ Sciences \\ Beijing, China
}

\begin{abstract}
Actual dynamic lift is an important index to measure the load capacity of agricultural unmanned aerial vehicle (AUAV). In real operation, it is difficult to obtain the accurate value of dynamic lift, which is easily affected by environmental factors, such as wind and other disturbances. In this paper, a test platform, induding hardware and software, has been developed. The main controller of the data acquisition system was PIC18F25K80, which could be used for PWM signal output and data communication. The experiment results showed that: the maximum mean absolute error of measurement system was less than $0.030 \mathrm{~kg}$. In addition, the pulse width of PWM signal was $1.0 \mathrm{~ms}$ when the rotator speed was $0 \mathrm{rad} / \mathrm{s}$, and then the rotator reached to the highest speed about $42.95 \mathrm{rad} / \mathrm{s}$ when pulse width equaled to $2.0 \mathrm{~ms}$. Moreover, lift coefficient $(k)$ of individual rotor to be tested was 0.003 , and the maximum load weight of individual rotor was about $5.377 \mathrm{~kg}$. The goodness of fit (R2) of curve which was obtained by the method of linearregression analysis was about 0.923 . This experimental study validated the effectiveness of dynamic lift model of AUAV rotor and laid the foundation for real-time dynamic lift measurement of AUAV.
\end{abstract}

Keywords- Agricultural Unmanned Aerial Vehicle (AUAV); Load Capacity; Rotor Dynamic Lift; Wireless Measurement and Control System; Rotator Speed

\section{INTRODUCTION}

Agricultural unmanned aerial vehicle (AUA V) is most advantageous for its low working altitude, simple structure, flexible, easy operation and extensive scope of application. It is increasingly popular in agricultural production, and its strength cannot be neglected particularly for the large-area information acquisition of farmland, spraying and fertilization, supplementary pollination or other agricultural tasks[1]. AUA V implements the action ability at 6 degrees of freedom (DOF) by controlling the lift change of its rotor and often need to carry a variety of sensors or operating equipment. To that regard, domestic and foreign scholars focally researches as to how to precisely obtain the relevant lift coefficient and examine the lift change in dynamic process of rotor. Farid Sharifi et al, deeply analy zed and measured the overall lift change of quadrotor UAV as interfered by the external environmental factor and enhances the fault-tolerant ability of this UA V by performing the sliding mode control[2]; Las zlo Kis et al, explored the control method of UAV safe landing in emergency, followed the dynamic change of lift in realtime and implemented the basic behavior control ability for the uncertain real system[3]; Liu Xuesong et al, asserted that mutual interference of rotors in multi-rotor UAV would create an effect on its overall lift[4]. By analyzing the diameter, chord length, variable pitch, rotor speed or other factors, they observed that smaller space between rotors means stronger interference and more loss of lift. In addition, as UAV normally operates at a lower flying speed, it is usually treated that the lift generated from the rotor is proportional to the square of rotor speed, namely constant lift will be generated when rotor operates at a certain speed[5]. In reality, however, different types of motors or rotors or other environmental factors might impact the dynamic lift of UAV. Further, some fluctuation of lift may arise from the lift change, followed by the 
disturbance of UAV control, which will lead to lessened precision of control, increased energy loss and more difficulty in determination of dynamic lift change in flight, along with the shortened lifetime of mechanical or electrical parts [6]. Thus, some real value can be identified as to the research of determination of dynamic lift for AUAV rotor with various types and structures.

This paper analyzes the dynamic lift model of AUA V rotor, establishes the rotator lift determination platform and dynamic lift measurement and control system and completes the dynamic lift determination of AUA V singleunit rotor accordingly. Moreover, it carries out the experiment and research works concerning the correlation among PWM signal duty ratio, rotator speed and rotor lift as well as determination method of dynamic lift coefficient.

\section{PRELIMINARIES}

\section{A. Model Analysis}

This paper analyses the dynamic lift change of AUAV by Euler-Lagrange formalism[7]. Take the quadrotor AUAV as an example, the barycentre of AUAV is the geometric center of body in ideal state. The parameters such as lift force, posture angle and direction should be calculated based on the reference frame. Consequently the Body reference frame and the Earth reference frame are built respectively [8]. The origin of Body reference frame is the geometric center of AUAV, as shown in Fig. 1.

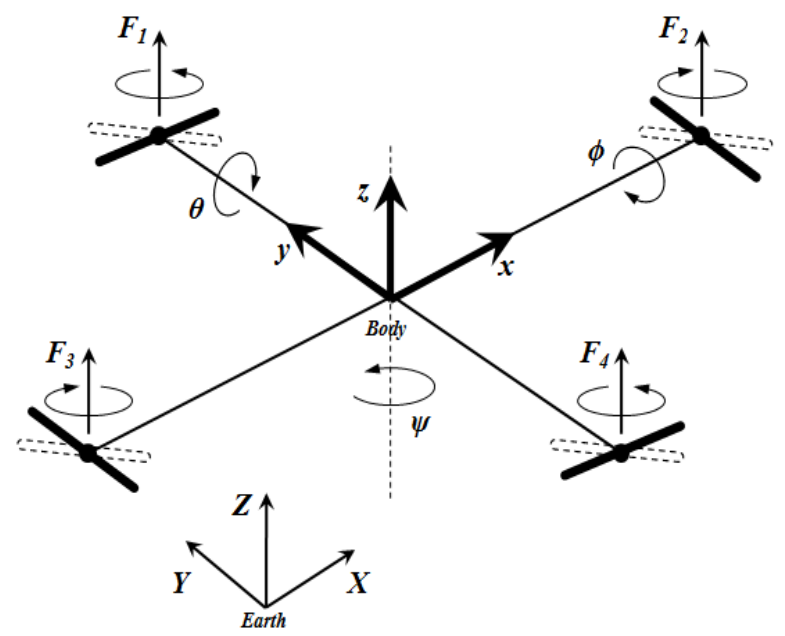

Figure 1. Dynamic lift model of quadrotor AUAV

In Fig. 1, the lift provided by AUAV rotator $i$ $(\mathrm{i}=1,2,3,4)$ is $\mathrm{F}_{\mathrm{i}}$ and in ideal state $F_{i}=k n_{i}^{2}$, where $\mathrm{k}$ is lift coefficient, and $n_{i}$ is the rotator speed[9]. It suggests that the lift coefficient $\mathrm{k}$ of each single rotator subject to the effect of many factors such as other rotors, environmental change and the posture of AUAV. Moreover, the parameters such as pitching, rolling and yaw are represented by vectors $(\theta, \phi, \psi)$. To definitely represent various dynamic parameters of AUAV and make it easier for the creation of force equation at each axis within Body reference frame, this paper implements the transformation of various parameters between Body reference frame and Earth reference frame by Euler angles and rotation matrix $\mathrm{R}_{\mathrm{EB}}[10]$.

$R_{E B}=$ $\left[\begin{array}{ccc}\cos \psi \cos \phi \quad \cos \psi \sin \theta \sin \phi \quad \cos \psi \sin \theta \cos \phi+\sin \psi \sin \phi \\ \sin \psi \cos \theta & \sin \psi \sin \theta \sin \phi & \sin \psi \sin \theta \cos \phi\end{array}\right.$

$\left[\begin{array}{ccc}\sin \psi \cos \theta & \sin \psi \sin \theta \sin \phi & \sin \psi \sin \theta \cos \phi-\sin \phi \cos \psi \\ -\sin \theta & \cos \theta \sin \phi & \cos \theta \cos \phi\end{array}\right]$ (1)

The correlation between overall lift and each rotator lift can be expressed by the Body reference frame of quadrotor AUAV:

$$
F_{B}=\left[\begin{array}{l}
F_{x B} \\
F_{y B} \\
F_{z B}
\end{array}\right]=\left[\begin{array}{c}
0 \\
0 \\
\sum_{i=1}^{4} F_{i}
\end{array}\right]
$$

The lift force of AUAV also can be expressed by equation (3) when combine with the Earth reference frame and rotation matrix $\mathrm{R}_{\mathrm{EB}}$ :

$$
\begin{aligned}
{\left[\begin{array}{l}
F_{x} \\
F_{y} \\
F_{z}
\end{array}\right]=} & R_{E B} \cdot F_{B}= \\
& \left(\sum_{i=1}^{4} k n_{i}^{2}\right)\left[\begin{array}{c}
\cos \psi \sin \theta \cos \phi+\sin \psi \sin \phi \\
\sin \psi \sin \theta \cos \phi-\sin \phi \cos \psi \\
\cos \theta \cos \phi
\end{array}\right]
\end{aligned}
$$

Therefore, based on the Earth reference frame, the equation of motion of quadrotor $\mathrm{AUAV}$ at $\mathrm{Z}$ axis can be expressed as:

$$
\begin{aligned}
& m \ddot{z}=F_{z}-m g-K \dot{z}= \\
& \quad\left(\sum_{i=1}^{4} k n_{i}^{2}\right)(\cos \theta \cos \phi)-m g-K \dot{z}
\end{aligned}
$$

where $\mathrm{K}$ is the resistance coefficient which mainly includes air resistance and mechanical resistance. It can be neglected at low rotator speed. Above all, the dynamic lift coefficient of AUAV is not only subject to the effect of rotor speed, but also relate to the parameters of AUAV such as pitching, rolling and yaw. Accurately measuring the dynamic lift coefficient of AUAV can effectively reflect its working state and assist to the precise control of AUAV movement.

\section{B. Design of Platform}

In order to accurate and comprehensive determine the dynamic lift coefficient of AUAV rotor and analyze the correlation of parameters among lift force, rotator speed and PWM signal duty ratio, this paper establishes the AUAV rotator lift determination platform based on the perspective of accuracy and safety of lift determination, and its specific structure is shown in Fig. 2.

The rotator lift determination platform mainly includes the non-contact rotational speed measurer, rotor, motor, electronic speed control (ESC), fixed frame, test platform, force sensor and computer measurement and control system. This paper selects the non-contact rotational speed measurer (DM6234) with measurement precision of $\pm 0.05 \%$ and force sensor (BK-2AS) with range of $0 \sim 20 \mathrm{~kg}$ and measurement precision of $\pm 0.05 \% \mathrm{~F}$.S for this platform. In addition, this rotator lift determination platform currently tests T-Motor multi-axis/rotor disk waterproof brushless motor (U11) and its carbon fiber rotor and FLAME 80A high-voltage ESC. This motor has the advantages of strong 
power, wide application and high energy efficiency and the rotational radius of rotor blade is about $36.83 \mathrm{~cm}$. This lift determination platform is not limited to the lift determination of single type motor or rotor. Because of the rotator lift direction of AUAV level upward in the process of actual flight, in order to facilitate the determination process and highly restore the real working condition of AUAV, this paper fixes the rotational speed measurer on the horizontal direction of the same plane of rotator which is apart from the test platform about $2 \mathrm{~m}$. This way could avoid the bigger error of measurement by holding the measurer in hand. This paper chooses the method which could convert the serial port to the wire less communication mode to read the data of force sensor or change the PWM signal duty ratio[11]. Then, the tester could complete the measurement and control work of lift force when they are far away from the high speed rotating AUAV rotor to ensure the security during in the experiment process.

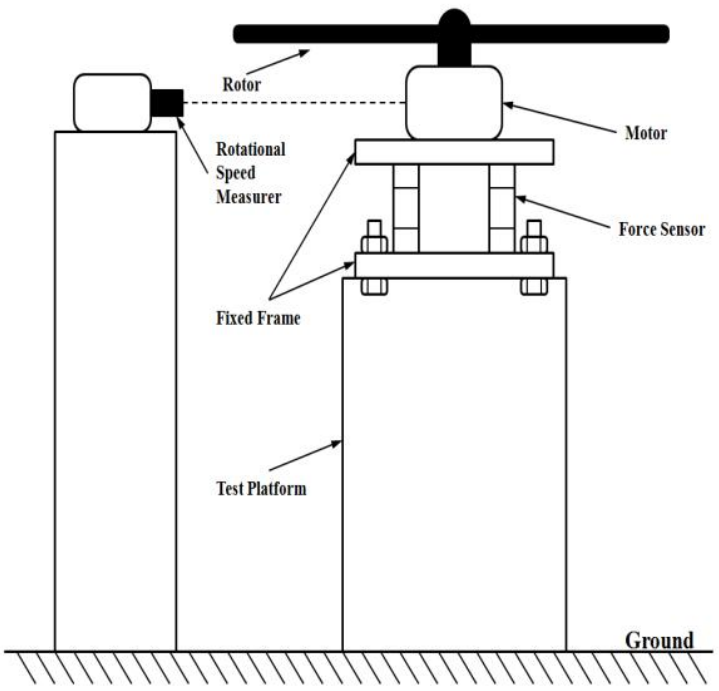

Figure 2. Structure diagram of rotator lift determination platform

\section{Design of Measurement and Control System}

Based on the above rotator lift determination platform, this paper designs and develops the AUAV rotor dynamic lift measurement and control system. The measurement and control system is mainly operated to drive and control the rotation of AUAV rotor and obtain the dynamic lift data accordingly. Its main feature is that increase the remote measurement and control device based on the rotator lift determination platform, namely computer software and bottom-layer information acquisition and control module. The concrete structure is shown in Fig. 3.

In Fig. 3, AUA V rotor dynamic lift measurement and control system is divided into the remote measurement and control device and rotator lift determination platform 2 parts. The computer software is primarily developed on VB platform in the remote measurement and control device. It could achieve the function which include the interconversion between sensor voltage and lift force, system calibration, data storage and PWM duty ratio adjustment. The information acquisition and control module is mainly composed by $\mathrm{PIC} 18 \mathrm{~F} 25 \mathrm{~K} 80$ chip, signal isolation and change-over circuit, serial communication, wireless communication and other external circuit. The PIC18F25K80 chip mainly consists of 4 PWM output, 4 timers and high-speed serial communication function and so on. It can replace the original receiver of AUA V to control ESC output by make a cycle of $20 \mathrm{~ms}$, duty ratio $5 \% \sim 10 \%$ of PWM signal according to the computer instruction. In addition, this measurement and control system adopts the effective isolation scheme for the output portion of information acquisition and control module. There are many advantages in this scheme such as reducing the external interference, preventing the situation of current overload from causing damage to the sensor, brushless motor and rotator lift determination platform. Besides, it also could enhance the practicability and stability of lift measurement and control system of AUAV. Remote Measurement and Control Device

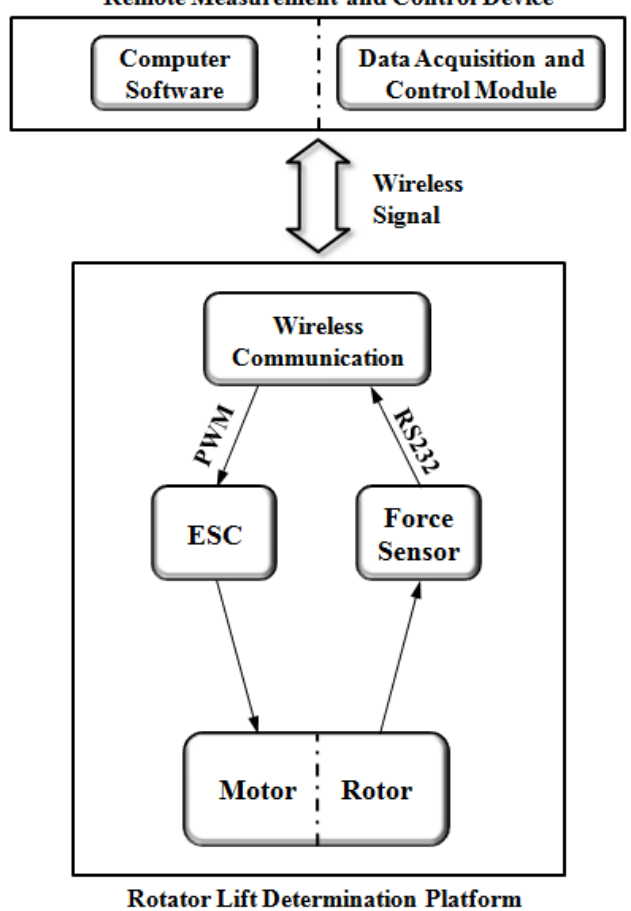

Figure 3. Block diagram of measurement and control system

\section{EXPERIMENT AL RESULTS}

\section{A. Calibration of Measurement and Control System}

In this paper, because of the rotator lift determination platform of UAV selects the force sensor BK-2AS as voltage output mode and independently designed and built the hardware of the measurement and control system, the calibration method is required to Figure out the quantitative relation between sensor output voltage and rotator lift. This calibration experiment firstly chooses 10 standard weights within $1 \mathrm{~kg} \sim 10 \mathrm{~kg}$ as the reference. Then, in order to highly reproduce the determination process of real lift force and reduce the system error, this paper puts the rotator lift determination platform of fixed frame upside down and additionally mounted a set of scale pans for placing these weights. This scale pan is fixed at the downward extended line of rotator center to keep the same way of the lift force which is provided by rotor. Using the computer software to get the net weight after the entire calibration system is stabilized. It could correspond to the voltage output of sensor and the real weight. This experiment of calibration is carried out under normal gravity environment, where $\mathrm{g}$ is $9.8 \mathrm{~N} / \mathrm{kg}$. The experiment is performed in 3 groups, and each of these groups starts 
calibration from $0 \mathrm{~kg}$. Adding $1 \mathrm{~kg}$ weight every time and repeating the test 11 times in total. By taking the average value of the 3 groups data and the Lagrange interpolation algorithm, this experiment Figure out the correlation between output voltage of force sensor and the corresponding load weight. The experiment contrasts the actual measurement value with calibration results, as shown in Fig. 4.

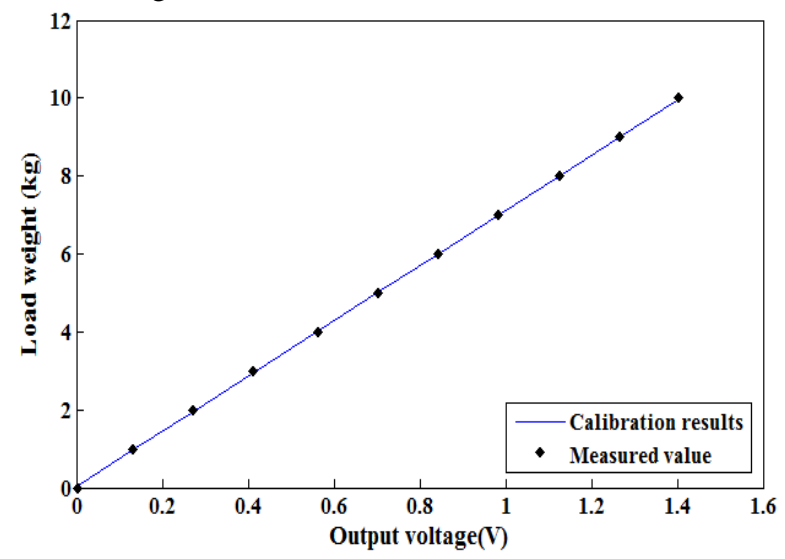

Figure 4. Calibration results of measurement and control system

According to Fig. 4, the calibration results show good linearity and only have smaller deviation with the actual measurement values. In order to obtain the accurate detection precision of rotator lift determination platform, this paper measures the standard weights by use the system which was calibrated. This experiment repeatedly tests for 5 times in every point and takes the average measurement value as for the final results, as shown in Table 1.

TABLE I. MEASUREMENT ACCURACY OF ROTATOR LIFT DETERMINATION PLATFORM

\begin{tabular}{|c|c|c|c|c|}
\hline \multirow{2}{*}{$\begin{array}{c}\text { Measuring } \\
\text { Point }\end{array}$} & \multicolumn{4}{|c|}{ Measurement Parameters } \\
\cline { 2 - 5 } & $\begin{array}{c}\text { Weight } \\
(\boldsymbol{k g})\end{array}$ & $\begin{array}{c}\text { Average } \\
\text { Measurement } \\
\text { Results }(\boldsymbol{k g})\end{array}$ & $\begin{array}{c}\text { Mean } \\
\text { Absolute } \\
\text { Error }(\boldsymbol{k g})\end{array}$ & $\begin{array}{c}\text { Standard } \\
\text { Deviation } \\
(\boldsymbol{k g})\end{array}$ \\
\hline 1 & 0 & 0.021 & 0.021 & 0.002 \\
\hline 2 & 1.000 & 1.030 & 0.030 & 0.001 \\
\hline 3 & 2.000 & 2.028 & 0.028 & 0.002 \\
\hline 4 & 3.000 & 3.027 & 0.027 & 0.001 \\
\hline 5 & 4.000 & 4.025 & 0.025 & 0.003 \\
\hline 6 & 5.000 & 5.027 & 0.027 & 0.003 \\
\hline 7 & 6.000 & 6.022 & 0.022 & 0.001 \\
\hline 8 & 7.000 & 7.020 & 0.020 & 0.001 \\
\hline 9 & 8.000 & 8.019 & 0.019 & 0.001 \\
\hline 10 & 9.000 & 9.021 & 0.021 & 0.002 \\
\hline 11 & 10.000 & 10.023 & 0.023 & 0.001 \\
\hline
\end{tabular}

In the experiment results of 11 times, by measure the standard weights, the experimental results showed that the maximum mean absolute error of force sensor was less than $0.030 \mathrm{~kg}$; the corresponding standard deviation $\sigma$ could be controlled within $0.001 \mathrm{~kg}$ to $0.003 \mathrm{~kg}$. It suggests that the rotator lift determination platform has high detection accuracy and stable detection result with lift force. This platform is able to meet the needs of the actual lift determination.

\section{B. Adjustment of Rotator Speed}

After calibrating the force sensor, the correspondence between duty ratio of PWM signal and rotator speed is tested and analyzed in this paper. Motor and carbon fiber rotor are mounted on the rotator lift determination platform, and the output cycle of control part in AUAV rotor lift measurement and control system is enabled $20.0 \mathrm{~ms}$ with PWM signal duty ratio of $5 \%$ to $10 \%$, namely the pulse width of PWM signal is controlled within $1 \mathrm{~ms} \sim 2 \mathrm{~ms}$. The corresponding rotator speed at the pulse width is measured after the stable rotating condition. This experiment is divided into 10 times, and each of them is repeated for 3 times to average the measurement values. As shown in Table 2.

TABLE II. CORRELATION OF PWM SIGNAL AND ROTATOR SPEED

\begin{tabular}{|c|c|c|}
\hline \multirow{2}{*}{ Test Point } & \multicolumn{2}{|c|}{ Test Parameters } \\
\cline { 2 - 3 } & $\begin{array}{c}\text { Pulse Width } \\
\text { of PWM Signal (ms) }\end{array}$ & Average Rotator Speed (rad/s) \\
\hline 1 & 1 & 0 \\
\hline 2 & 1.2 & 3.02 \\
\hline 3 & 1.3 & 8.87 \\
\hline 4 & 1.4 & 14.45 \\
\hline 5 & 1.5 & 20.02 \\
\hline 6 & 1.6 & 26.97 \\
\hline 7 & 1.7 & 32.13 \\
\hline 8 & 1.8 & 37.00 \\
\hline 9 & 1.9 & 41.17 \\
\hline 10 & 2.0 & 42.95 \\
\hline
\end{tabular}

According to Table 2, the rotator speed increases with PWM signal duty ratio under the condition of load, the pulse width of PWM signal is $1.0 \mathrm{~ms}$ when the rotator speed is $0 \mathrm{rad} / \mathrm{s}$, and then the rotator reach to the highest speed about $42.95 \mathrm{rad} / \mathrm{s}$ when pulse width equal to $2.0 \mathrm{~ms}$. When the windward area of rotor and air density remains unchanged, the air resistance increases with the rotor speed in non-linear relationship. For that reason, although the increase of the pulse width has not changed, the increase rate of the rotator speed gradually decreases in 7 to 10 times tests. When the pulse width is greater than $2 \mathrm{~ms}$, the rotator speed which is remained at maximum speed will not increase with the pulse width of PWM signal.

\section{Determination of Dynamic Lift Coefficient}

Besides the correlation between PWM signal duty ratio and rotator speed, the influence of the rotator speed and the dynamic lift coefficient on the actual load capacity of AUAV is analyzed in this paper. The experiment is conducted under the condition of indoor environment without wind, and it is mainly divided into 3 steps:

- Getting the net weight by computer software after the lift determination platform and entire measurement and control system are stabilized. Then begin to measure the actual lift of the rotor. 
- The output scheme of duty ratio of control system is kept the same way to the above section. The rotator speed and the corresponding lift are recorded in the process of rotor dynamic rotating. This experiment is divided into 10 groups, and each group is repeated for 3 times to Figure out the average value.

- The PWM signal duty ratio output in the reverse way by the original mode, and then repeat Step 2 and record the experimental data.

The experimental results are shown in Fig. 5.

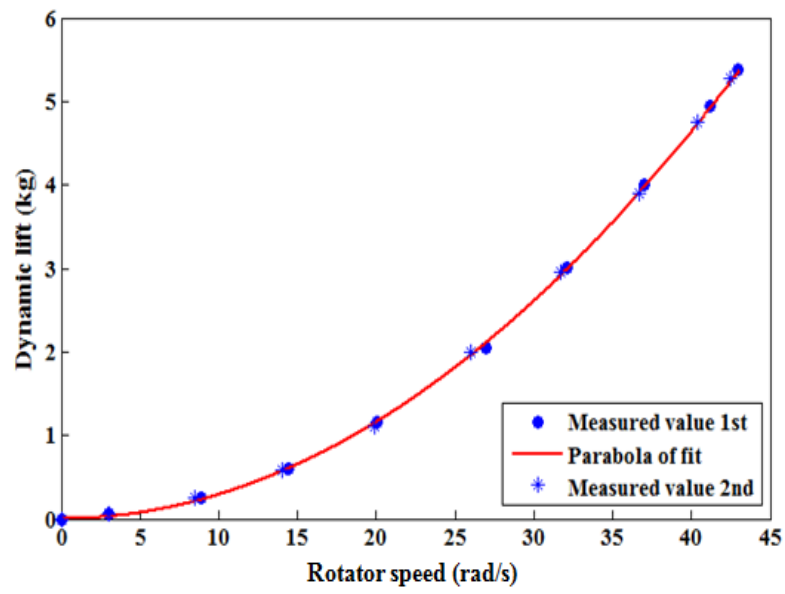

Figure 5. Correlation of rotator speed and dynamic lift

According to Fig. 5, the lift of rotor increases with rotator speed but not perform strict linear relationship. In the same duty ratio, the first actual speed is slightly higher than the second. This is because the hysteresis characteristic of force sens or and the long-time working which could cause the reducing of battery voltage. By analyzing the dynamic lift model of AUAV rotor, the parabola $\mathrm{y}=\mathrm{kx}^{2}$ is used to fit the measurement values for the red curve which is showed in Fig. 5. The fitting results basically coincide with two actual measurement values. By the method of linear-regression analysis, the goodness of fit $\mathrm{R}^{2}$ of fitting curve which is about 0.923 is obtained in this experiment. It means the fitting curve has good prediction effect. Furthermore, because the measurement values of force sens or are the weight rather than force, this paper converts the actual weight to the lift force by the equation (5).

$$
F=m g=k n^{2} g
$$

where, $\mathrm{F}$ is the lift force of AUA V individual rotor, $\mathrm{n}$ is the rotator speed, $g=9.8 \mathrm{~N} / \mathrm{kg}$ (gravity constant), and $\mathrm{k}=0.003$. This experiment showed that: the dynamic lift coefficient $(\mathrm{k})$ of motor and rotor to be tested is 0.003 in the condition of indoor environment; the maximum load weight of individual rotor is about $5.377 \mathrm{~kg}$; the actual dynamic lift of rotor is proportional to the square of rotator speed, and it can be approximately expressed with the equation (5).

\section{CONCLUSIONS}

This paper carefully analyzed the dynamic lift model of AUAV and established the rotator lift determination platform and dynamic lift measurement and control system accordingly. It chose the T-motor brushless motor (U11) and carbon fiber rotor for the actual research object and implemented the function of rotor speed control and the determination of dynamic lift by using the remote measurement and control device and various types of sensors. The following conclusions are obtained by experiments:

- The calibration experiment of the AUAV rotor dynamic lift measurement and control system was accomplished. The calibrated results showed good linearity and only had smaller deviation with the actual measurement values. The experiment showed that the maximum mean absolute error of measurement system was less than $0.030 \mathrm{~kg}$. The corresponding standard deviation $\sigma$ could be controlled within $0.001 \mathrm{~kg}$ to $0.003 \mathrm{~kg}$. It suggested that the rotator lift determination platform had high detection accuracy and was able to meet the needs of the actual lift determination.

- The correspondence between duty ratio of PWM signal and rotator speed is tested and analyzed in this paper. The output cycle of measurement and control system was enabled $20.0 \mathrm{~ms}$ with PWM signal duty ratio of $5 \%$ to $10 \%$. The measured motor could accomplish the variable process which contained the minimum speed 0 to the highest speed 42.95rad/s. The rotator speed increased with PWM signal duty ratio under the condition of load.

- This paper completed the determination of dynamic lift coefficient for AUAV. The experiment was conducted under the condition of indoor environment without wind, and results showed that the lift coefficient $(\mathrm{k})$ of individual rotor to be tested was 0.003 ; the maximum load weight of individual rotor was about $5.377 \mathrm{~kg}$. The actual dynamic lift of rotor was proportional to the square of rotator speed, and the goodness of fit $R^{2}$ of curve which was obtained by the method of linear-regression analys is was about 0.923 . This experiment and research validated the effectiveness of dynamic lift model of AUAV rotor and laid the foundation for real-time dynamic lift measurement of AUAV.

\section{ACKNOWLEDGMENT}

The work by Zhang Jing, Chen Du, Wang Shumao, Wei Liguo and Jia Quan was supported in part by the Opening Fund of State Key Laboratory of Soil Plant Machinery System Technology (2014-SKL-01) and Research Fund from Beijing Key Laboratory of Optimal Design for modern Agricultural Equipment (D151100003715001).

\section{REFERENCES}

[1] G.M. Jahangir Alama, Md. Mamunb, Md. Abu. Taher Alib, et al, "Investigation of the aerodynamic characteristics of an aerofoil shaped fuselage UAV model," Procedia Engineering, vol. 90, Nov. 2014, pp. 225-231.

[2] Farid Sharifi, Mostafa Mirzaei, Brandon W, et al, "Fault Tolerant Control of a Quadrotor UAV using Sliding Mode Control," Conference on Control and Fault Tolerant Systems, Oct. 2010, pp. 239-244. 
[3] Laszlo Kis and Bela Lantos, "Quadrotor Control Based on Partial Sensor Data," 19th International Workshop on Robotics, Jun. 2010, pp. 43-48.

[4] Liu Xuesong, Ang Haisong and Xiao Tianhang, "Analysis of Rot or Interference Effects on Quad-rotor Lift in Hover," ADVANCES IN AERONAUTICAL SCIENCE AND ENGINEERING, vol. 5, No. 2, May. 2014, pp. 148-153.

[5] Tan J F, Wang H W and Lin C L, "Analysis of influence of rotor parameters on rotor hover performance by lifting-surface and free wake method," Acta Aeronautica et Astronautica Sinica, vol. 33, No. 2, Feb. 2012,pp. 249-257.

[6] Li Jiyu, Zhou Zhiyan, Hu Lian, et al, "Optimization of operation parameters for supplementary pollination in hybrid rice breeding using uniaxial single-rotor electric unmanned helicopter," Transactions of the Chinese Society of Agricultural Engineering, vol. 30, No. 10, May. 2014,pp. 11-17.

[7] Zhao Changjun, Gong Xun, Bai Yue, et al, "Interference and inhibition of lift fluctuation on quadrot or aircraft," Optics and Precision Engineering, vol. 22, No. 9, Sept. 2014, pp. 2431-2437.
[8] Prat ap Tokekar, Joshua Vander Hook, David Mulla, et al, "Sensor Planning for a Symbiotic UAV and UGV system for Precision Agriculture," IEEE/RSJ International Conference on Intelligent Robots and Systems, Nov. 2013, pp. 5321-5326.

[9] I.S. Kry vokhat ko and V.V. Sukhov, "Experimental Investigation of Aerodynamic Performance of a Small UAV With a Telescopic Wing," Proc. IEEE Symp. Actual Problems of Unmanned Air Vehicles Developments, 2013, pp. 17-20.

[10] Yizhe Zhang, Junyi Zuo and Bingrong Zhang, "Modularized Modeling and Inversed Dynamics Control Design for a Lift-fan UAV in Hover," Proc. IEEE Symp. International Conference on Information and Aut omation, Aug. 2013, pp. 570-575.

[11] Zhang Xing, Bai YongQiang, Xin Bin, et al, "Differential Evolution Based Receding Horizon Control for UAV Motion Planning in Dynamic Environments," ROBOT, vol. 35, No. 1, Jun. 2013, pp. 98-107. 